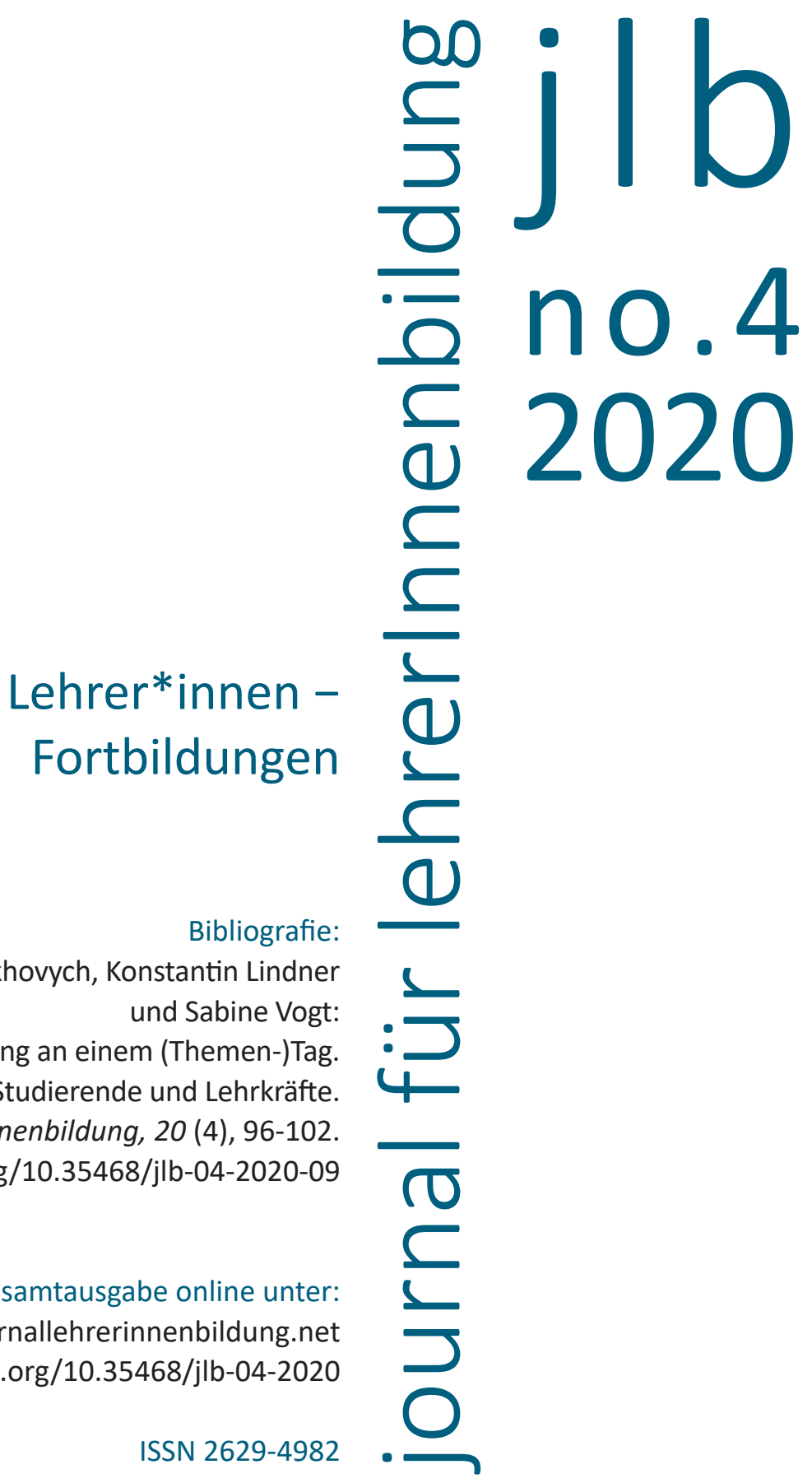


09

Adrianna Hlukhovych, Konstantin Lindner und Sabine Vogt

\section{Kulturelle Bildung an einem (Themen-)Tag. Ein Format für Studierende und Lehrkräfte}


Geistes- und kulturwissenschaftliche Zugänge haben für die Deutung der Welt, für die Entfaltung von Kommunikations- und Handlungskompetenzen sowie für die kulturelle Weiterentwicklung hohe Relevanz (Bauer, Beuter, Lindner, Hlukhovych \& Vogt, 2018). Als Teilprojekt des 2016 bis $2023 \mathrm{im}$ Rahmen der „Qualitätsoffensive Lehrerbildung“ an der Otto-Friedrich-Universität Bamberg geförderten BMBF-Großprojektes „WegE: Wegweisende Lehrerbildung - Entwicklung reflexiver Kommunikationsprozesse“ bündelt das Projekt „KulturPLUS: Kulturbezogene Bildung" disziplinenübergreifend Kompetenzen der geistes- und kulturwissenschaftlichen Fachwissenschaften und Fachdidaktiken und macht sie für die Lehrer*innenbildung und Lehrer*innenfortbildung fruchtbar. Dazu dienen nicht nur Vorlesungen und Seminare, die vor allem von Studierenden im Rahmen ihrer Studiengänge belegt werden (es wurden dazu eigens KulturPLUS-Module in den Lehramtsstudiengängen implementiert), sondern auch das neu konzipierte Format eines "Thementages", das sich an Lehramtsstudierende sowie Lehrkräfte richtet und im Folgenden vorgestellt wird.

\section{Das inhaltliche Konzept: Kultur und kulturelle Bildung}

Kultur umfasst den von Menschen erzeugten Gesamtkomplex von Vorstellungen, Denkformen, Empfindungsweisen, Werten und Bedeutungen, der sich in Symbolsystemen niederschlägt. Die jeweiligen Symbolsysteme greifen auf spezifische Formen der Weltinterpretation zurück. Sie entstehen, werden reproduziert und verändern sich (Nünning, 2009). Als Sinn- und Unterscheidungssysteme sind sie kein bloßer gesellschaftlicher „Überbau“, sondern sie beteiligen sich an der Organisation und Interpretation der Wirklichkeit (Reckwitz, 2004, S. 7). Welt existiert somit als vom Menschen mit Bedeutungen versehene und interpretierte. Um sie verstehen, deuten und gestalten zu können, um mit dem immerwährenden Wandel von Kulturen und Kulturauffassungen mithalten zu können, bedarf es - vor allem im Bildungskontext und damit auch in der Lehrer*innenbildung - entsprechender nachhaltiger Maßnahmen und Formate, die auf Aktualität einerseits und lebenslanges Lernen andererseits sowie auf (Selbst-) Reflexion ausgerichtet sind. Diese Maßnahmen und Formate sollten im Sinne des Auftrags kultureller Bildung - ermöglichen: (1) vielfältige 
kulturelle Ordnungen und Codierungsformen in der Welt wahrzunehmen, sie zu deuten bzw. sie zu decodieren, (2) sich selbst in der Welt zu verorten bzw. sich in dem „selbstgesponnenen“ kulturellen „Bedeutungsgewebe" (Geertz, 2012 [1973], S. 9) zurechtzufinden und die eigene Positionierung zu reflektieren sowie (3) kulturelle Codierungen vorzunehmen bzw. das eigene Leben und die Welt zu gestalten.

\section{Das didaktische Konzept: KulturPLUS-Thementage}

Um einen Beitrag zur kulturellen Lehrer*innen(fort)bildung im Sinne eines solchen weitgefassten Kulturbegriffs zu leisten, hat das Projekt KulturPLUS Lehrveranstaltungen zu kulturellen bzw. kulturbildenden Themen initiiert, die Fach- und Disziplingrenzen überschreiten und von aktueller gesellschafts- und bildungspolitischer Relevanz sind: Ringvorlesungen und insbesondere Thementage zu Themen wie „Europa“, "Sprachen“, „Kulturelles Gedächtnis“, „Materielle Kulturen“, „Migration“, „Die Macht der Bilder“.
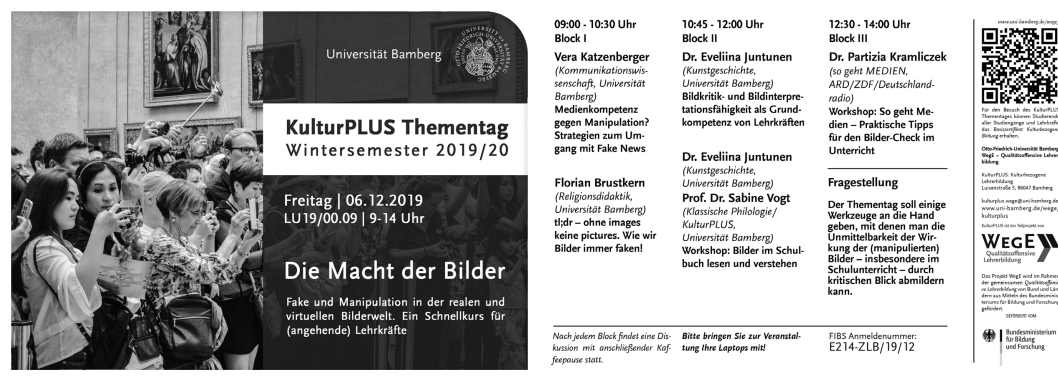

Abb. 1 KulturPlus-Thementag

Studierende und Lehrkräfte aller Schularten und Fachrichtungen erhalten hierdurch die Möglichkeit, über ihre eigenen Studiengänge und Fachrichtungen hinaus Einblicke in grundlegende kulturelle Themenbereiche und Fragestellungen zu gewinnen und diese in Bezug auf die Verzahnung von Theorie und Unterrichts- sowie außerschulischer Praxis zu reflektieren. Zu diesem Zweck werden Expert*innen aus unterschiedlichen Bereichen eingeladen, die eine professionelle fachliche Basis der Thementage auf der inhaltlichen Ebene gewähr- 
leisten (Lipowsky, 2010, S. 40) und zu deren Wirksamkeit beitragen (Lipowsky, 2010, S. 64): Wissenschaftler*innen der Universität Bamberg und anderer Hochschulen, Lehrkräfte, Journalist*innen sowie Vertreter*innen kultureller und Bildungseinrichtungen wie Theater, Museen, Religionsgemeinschaften, Medieninstitutionen, kultur- und bildungsrelevanter Behörden oder Nichtregierungsorganisationen.

Ein Thementag zeichnet sich durch seine multiperspektivische und verschiedene Kontexte vernetzende Konzeption aus: Lehramtsstudierende und aktive Lehrkräfte arbeiten im Austausch mit Expert*innen an Fragen kultureller Bildung. Dies geschieht in drei Blöcken à ca. 120 Minuten, die didaktisch unterschiedlich gestaltet werden: Impulsvorträge wechseln sich ab mit Berichten aus der Praxis, Expert*innengesprächen und Workshops oder Kleingruppenarbeit, und es gibt ausreichend Gelegenheit zum Erfahrungsaustausch und zur Diskussion. Unter anderem diese strukturelle Vielfalt von Methoden und Lerngelegenheiten macht den Erfolg der Thementage aus (Richter, 2016).

Die Referent*innen werden gebeten, bei der Vorbereitung ihrer Beiträge folgende Leitfragen zu berücksichtigen:

- Exemplarische Verortung des Beitrags: Wo bzw. wie kommen Schüler*innen in ihrer „Lebenswelt" mit dem Thema in Berührung?

- Fachspezifische Erschließung: Welche Theorien, Forschungsergebnisse oder Praxiskontexte der jeweiligen Fachdisziplin sind für die Fragestellung des Beitrags bzw. für deren Vermittlung an (angehende) Lehrkräfte relevant?

- Inhaltliche Impulse für kulturelle Lehrer*innenbildung: Warum und hinsichtlich welcher Aspekte ist der Beitrag für künftige Lehrkräfte von Bedeutung? Welche Konzepte sollten (angehenden) Lehrkräften mitgegeben werden, die sich mit der Thematik in ihrem Lehramtsstudiengang oder beruflichen Alltag nicht (oder nicht vertieft) beschäftigen?

- Praktische Impulse für kulturelle Lehrer*innenbildung: Welche konkreten Umsetzungsoptionen zur Erschließung des Themas mit Schüler*innen können mitgegeben werden, damit sich die Teilnahme an Thementagen in verbesserten Lernzugewinnen der Schüler*innen manifestiert (Richter, 2016)? Welche weiterführenden Informationen (Sekundärliteratur, Schulungsmaterialien, Internetressourcen etc.) zur eigenständigen Weiterbildung können empfohlen werden? 
Denn „[d]er in den Fortbildungen angestoßene Lernprozess soll dazu beitragen, die bestehende berufliche Praxis zu reflektieren, sowie neue Erkenntnisse und Kompetenzen in die Gestaltung des Unterrichts einzubringen". Das heißt, die Fortbildung am Thementag soll sich nicht nur auf die Kompetenzen von Lehrkräften auswirken, sondern auch im beruflichen Handeln „und in der Arbeit mit Schülerinnen und Schülern und ihren Lernergebnissen sichtbar werden" (Richter, 2016, 253). Auf diese Weise erfüllen Thementage den Anspruch aller Fortbildungen, ein effektives Instrument zur Verbesserung der Lehrer*innenprofessionalität zu sein (Lipowsky \& Rzejak, 2015).

\section{Perspektiven \\ für die kulturelle Lehrer*innenbildung}

Das Format des KulturPLUS-Thementags entspricht dem eingangs skizzierten Auftrag kultureller Bildung in vielerlei Hinsicht: Es ist ein kompaktes und zugleich ein arbeits- und reflexionsintensives Format, das dem Tempo der gegenwärtigen Lebensrealität und dem Modus verschiedener kultureller und gesellschaftlicher Transformationsprozesse entspricht. An einem Tag werden die Teilnehmenden mit einem aktuellen kulturellen Thema bzw. Phänomen sowie mit dessen Folgerungen und Herausforderungen für den schulischen Kontext in gebündelter Form vertraut gemacht. Unter anderem sorgen die eintägige Intensität, Kompaktheit wie seine Terminierung an Freitagen für hohe Teilnehmer*innenzahlen seitens der Studierenden, vor allem aber von Lehrkräften, die sich für ganztägige Formate eher Freiräume schaffen können als für regelmäßig wöchentlich stattfindende universitäre Formate wie Seminare oder (Ring-)Vorlesungen, was zur Vereinbarkeit von beruflichen Aufgaben und Fortbildungsteilnahme beiträgt (Richter, 2016).

Dank der interdisziplinären Ausrichtung der Thementage sowie der Beteiligung von Referent*innen aus unterschiedlichen Disziplinen und Praxiskontexten werden die Themen aus diversen spezifischen Deutungs- bzw. Decodierungs-Perspektiven - eben im Sinne von Kultur als Gesamtkomplex von Vorstellungen, Denkformen, Empfindungsweisen, Werten und Bedeutungen - beleuchtet. Dabei treten Fachwissenschaften und Fachdidaktiken, Wissenschaft und Praxis in Dialog; 
theoretische Ansätze werden - im Sinne eines aktiven Lernens (Richter, 2016) und der Partizipation (Lipowsky, 2010) - didaktisch operationalisiert und in praktischen Workshops erprobt. Die Kombination aus Reflexions- und handlungspraktischen Phasen sichert die Wirksamkeit der Fortbildung und den Transfer in die Unterrichtspraxis (Lipowsky, 2010). Das Thementag-Format bietet den Teilnehmenden die Gelegenheit, ihre Verortung im kulturellen Gesamtkomplex zu reflektieren sowie die Gestaltung kultureller Prozesse zu üben. Die Vielfalt der Perspektiven und (Selbst-)Reflexionsmöglichkeiten wird bereichert durch den Austausch unter den Referierenden, vor allem aber unter (Lehramts-)Studierenden und Lehrkräften, was den Wissenstransfer zwischen unterschiedlichen Phasen der Lehrer*innenbildung und den Lernerfolg (Lipowsky, 2010) unterstützt. Im Sinne der Nachhaltigkeit können die Teilnehmer*innen der Thementage auf die (weiterführenden) Materialien zum Thementag in Moodle zurückgreifen. Ergänzende Online-Formate, die Synergien zwischen synchronen und asynchronen Lehrformaten und Austauschmöglichkeiten schaffen, sind für die Zukunft geplant. ${ }^{1}$

\section{Literatur}

Bauer, B., Beuter, K., Lindner, K., Hlukhovych, A. \& Vogt, S. (2018). Kulturbezogene Lehrerinnen- und Lehrerbildung. Grundlegungen, Perspektiven und Operationalisierungsoptionen. In A. Hlukhovych, B. Bauer, K. Beuter, K. Lindner \& S. Vogt (Hrsg.), Kultur und kulturelle Bildung. Interdisziplinäre Verortungen - Lehrerinnenund Lehrerbildung - Perspektiven für die Schule (S. 13-36). Bamberg: UBP.

Geertz, C. (2012 [1973]). Dichte Beschreibung. Beiträge zum Verstehen kultureller Systeme. Frankfurt am Main: Suhrkamp.

Lipowsky, F. (2010). Lernen im Beruf. Empirische Befunde zur Wirksamkeit von Lehrerfortbildung. In F. H. Müller, A. Eichenberger, M. Lüders \& J. Mayr (Hrsg.), Lehrerinnen und Lehrer lernen. Konzepte und Befunde zur Lehrerfortbildung (S. 51-70). Münster: Waxmann.

Lipowsky, F. \& Rzejak, D. (2015). Key features of effective professional development programmes for teachers. RICERCAZIONE. Six-monthly Journal on Learning Research and Innovation in Education, 7(2), 27-51.

1 Das diesem Artikel zugrundeliegende Vorhaben wird im Rahmen der gemeinsamen „Qualitätsoffensive Lehrerbildung" von Bund und Ländern mit Mitteln des Bundesministeriums für Bildung und Forschung unter dem Förderkennzeichen 01JA1615 gefördert. Die Verantwortung für den Inhalt dieser Veröffentlichung liegt bei den Autorinnen und dem Autor. 
Nünning, A. (2009). Vielfalt der Kulturbegriffe. Verfügbar unter https://www.bpb.de/ gesellschaft/bildung/kulturelle-bildung/59917/kulturbegriffe? $p=a l l[12.09 .2020]$.

Reckwitz, A. (2004). Brennpunkte einer kulturwissenschaftlichen Interpretation der Kultur. In F. Jaeger \& J. Rüsen (Hrsg.), Handbuch der Kulturwissenschaften. Bd. 3: Themen und Tendenzen (S. 1-20). Stuttgart: Metzler.

Richter, D. (2016). Lehrerinnen und Lehrer lernen: Fort- und Weiterbildung im Lehrerberuf. In M. Rothland (Hrsg.), Beruf Lehrer/Lehrerin: Ein Studienbuch (S. 245-260). Stuttgart: UTB.

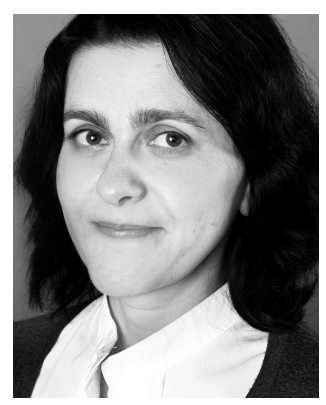

Adrianna Hlukhovych, Dr., wiss. Mitarbeiterin/Koordinatorin im Projekt KulturPLUS/WegE, Otto-Friedrich-Universität Bamberg. Arbeitsschwerpunkt: Konzepte kultureller Bildung aus kultur-/medienwissenschaftlicher Sicht

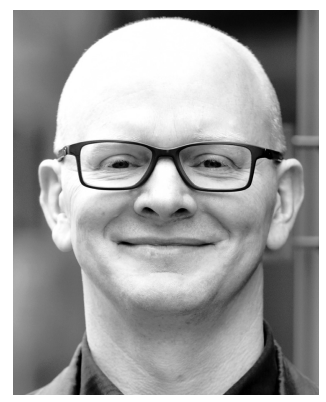

Konstantin Lindner, Dr., Professor für Religionsdidaktik, Otto-Friedrich-Universität Bamberg. Sprecher des Projekts KulturPLUS/WegE. Arbeitsschwerpunkte: Kulturelle (Lehrer*innen-)Bildung, kooperativer Religionsunterricht, Wertebildung

\section{konstantin.lindner@uni-bamberg.de}

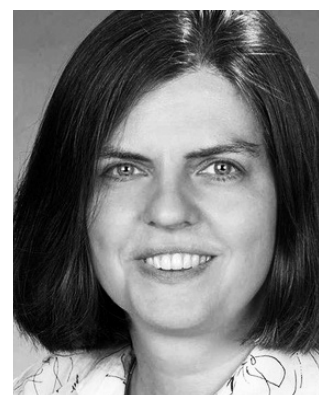

Sabine Vogt, Dr., Professorin

für Klassische Philologie/Gräzistik, Otto-Friedrich-Universität Bamberg. Sprecherin des Projekts KulturPLUS/WegE. Arbeitsschwerpunkte: Kulturelle (Lehrer*innen-)Bildung, transdisziplinäre Vermittlung der Antike

sabine.vogt@uni-bamberg.de 